\title{
A plasticity theory approach for the stability analysis of vertical layers of concrete in the fresh state
}

\section{Citation:}

Torelli G, Lees JM (2019) A plasticity theory approach for the stability analysis of vertical layers of concrete in the fresh state. In: Proceedings of the 2nd International RILEM Conference on Rheology and Processing of Construction Materials (RheoCon2) and the 9th International RILEM Symposium on Self-Compacting Concrete (SCC9). Dresden (Germany).

\section{Version:}

Accepted for publication. 


\title{
A plasticity theory approach for the stability analysis of vertical layers of concrete in the fresh state
}

Giacomo Torelli ${ }^{1}$, Janet M. Lees ${ }^{1}$

${ }^{1}$ University of Cambridge, Cambridge, United Kingdom

\begin{abstract}
The industrial production of cement is currently responsible for around $5 \%$ of global $\mathrm{CO}_{2}$ emissions. Hence, the development of technologies aimed at minimizing the use of cement in concrete structures, while preserving their strength and durability properties, plays a vital role in the reduction of carbon emissions.

The use of cement in concrete structures can be minimized through the manufacture of functionally layered structural elements where concrete with high cement content is used rationally only when it contributes significantly to the performance of the structure. In functionally layered concrete, horizontal variation in material composition can be achieved by casting adjacent vertical layers of different materials. Removable vertical panels can be used to demarcate the mixes during casting. A good bond between the layers can be achieved by removing the panels prior to concrete hardening. However, a major problem with this application is the control of the fresh-state deformations of the adjacent vertical layers.
\end{abstract}

This study investigates the fundamental problem of fresh state stability of concrete prisms that consist of two vertical layers of different mixes. A novel limit-state approach based on plasticity theory is formulated to assess the stability of the system as a function of material properties and geometry. The relationship between material parameters, system stability and geometry is determined and the formulated limit-state approach is validated against experimental results.

Keywords: Concrete; stability; plasticity; rheology; limit-state analysis

\section{Introduction}

Concrete is the most widely used construction material in the world. The manufacture of cement, the key constituent of concrete, is energy intensive and accounts for around $5 \%$ of global $\mathrm{CO}_{2}$ emissions related to human activities [1]. Minimizing the use of cement in concrete structures is thus a great opportunity to reduce the global $\mathrm{CO}_{2}$ emissions.

The use of cement can be minimized by manufacturing functionally layered concrete elements, i.e. concrete elements composed of various layers of different concrete mixes. In this way, mixes with high cement content can be used only where they are needed. The material composition of concrete elements is commonly varied in the vertical direction by 
sequentially casting horizontal layers of different concrete mixes. Nevertheless, relatively little attention has been paid to the possibility of varying the material composition in the horizontal direction by casting adjacent vertical layers of different mixes. This concept represents a clear opportunity to further optimize the use of cement in structural elements such as beams, columns and walls. If, for example, a relatively low water/cement ratio is needed in a vertical concrete cover to minimize porosity and maximize durability, concrete with high cement content can be used only in that region.

However, the major obstacle in the implementation of this concept is the control of the fresh state deformations of the vertical layers. Indeed, if the mixes are fluid enough, global instability phenomena can occur following the removal of a panel where heavier mixes tend to flow underneath lighter mixes. As a consequence, the desired spatial variation in concrete composition may be compromised. Accurate understanding of the parameters driving the fresh state instability of vertical layers of concrete is thus needed.

This paper presents an original analytical method to investigate the fundamental problem of the fresh state stability of two adjacent vertical layers of different concrete mixes (see Figure 1). Two limit-state models, based on the upper and lower bound theorems of plasticity are developed to capture the relationship between system geometry, material parameters and the stability of the system. The newly formulated analysis method is validated and discussed in light of experimental results.

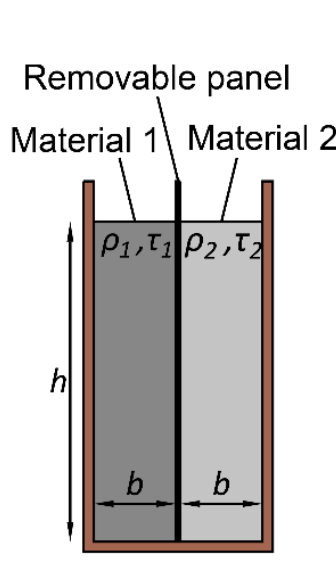

a)

Casting

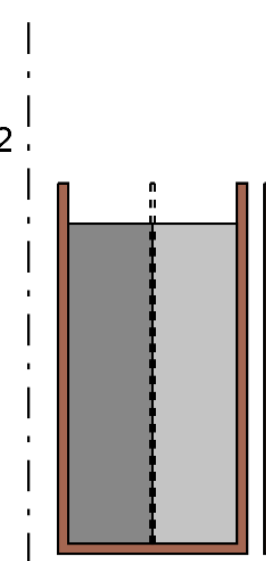

b.1)

Stable

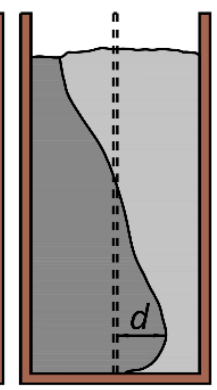

b.2)

Unstable

Figure 1. a) Casting, b.1) stable fresh state behavior, and b.2) unstable fresh state behavior of an element composed of two vertical layers of different mixes.

\section{Modelling approach}

Fresh cementitious materials are commonly modelled as yield stress fluids. That is, they are assumed to behave as solids for low stresses and to flow when a threshold shear stress, 
referred to as yield stress $\tau$, is exceeded. Experimental flow curves relating shear stress and shear rate can be approximated by a Herschel-Bulkley model:

$$
\tau>\tau_{0} \rightarrow \tau=\tau_{0}+k \dot{\gamma}^{n}
$$

where $\tau_{0}$ is the yield stress, $\dot{\gamma}$ is the shear rate, $k$ is the consistency index, and $n$ is the flow index. The material parameters $\tau_{0}, k$ and $n$ can be controlled by selecting the appropriate mix constituents and their relative proportions [2-5].

In this work it is assumed that, when the yield stress is exceeded, flow occurs at small shear rates. From Equation 1 it can be seen that for small shear rates the shear stress tends to $\tau_{0}$. Under these assumptions, the behaviour of the material is modelled through the theory of perfect plasticity. An exact analysis of a plastic body would require that equilibrium, compatibility and constitutive equations be satisfied simultaneously [6-8]. An alternative to a full plastic analysis is to focus on the stability limit state. This approach is justified when phenomena occurring prior to collapse are not of interest. The stability limit state of a plastic body can be studied through the upper and lower bound methods of plasticity theory [7,9]. The upper bound method allows an upper bound of the exact collapse load to be determined by ignoring the equilibrium conditions. By contrast, the lower bound method allows a lower bound of the exact collapse load to be determined by ignoring the compatibility conditions.

Two models, based on the upper and lower bound theorems of plasticity, are formulated here to assess the relationships between material properties, geometry, boundary conditions and stability of the system presented in Figure 1.

\section{Plastic models}

\subsection{Upper bound model}

An upper bound model is developed that assumes the activation of the compatible mechanism shown in Figure 2a. The kinematic model consists of four rigid blocks $(A, B$, $E, D)$ and two radial shear zones $\left(C_{1}\right.$ and $\left.C_{2}\right)$. In such a mechanism a heavier material would flow underneath a lighter material. In line with the definition of the model, it is assumed that the shear stress developing on slip surfaces between blocks is the yield stress of the material. At the walls, a fraction $f$ of the yield stress of the material is considered, while the minimum yield stress of the two materials is assumed to develop at the interface between the materials. Equating external and internal work for the mechanism reported in Figure 2a, leads to:

$$
\tau_{s}=m_{U} \Delta \rho
$$


where $\tau_{s}$ is the sum of the yield stresses $\tau_{1}$ and $\tau_{2}$ of material 1 and 2 respectively, $\Delta \rho$ is the difference in density between the two materials and $m_{U}$ is here defined as upper-bound stability coefficient:

$$
m_{U}=\frac{g}{k_{U 1}+k_{U 2}+k_{U 3}}
$$

where $g$ is the gravitational acceleration and $k_{U 1}, k_{U 2}$, and $k_{U 3}$ are:

$$
k_{U 1}=\frac{\left(2+\frac{\pi}{2}\right) b}{b h-\frac{\sqrt{2}}{4} b^{2}} ; \quad k_{U 2}=\frac{f\left(h-\frac{b}{\sqrt{2}}\right)}{b h-\frac{\sqrt{2}}{4} b^{2}} ; \quad k_{U 3}=\frac{\frac{2 \alpha}{\alpha+1}\left(h-\frac{b}{\sqrt{2}}\right)}{b h-\frac{\sqrt{2}}{4} b^{2}}
$$

where $b$ is the width of the column, $h$ the height of the system and $\alpha$ is defined as the ratio between the smaller and the larger of the two yield stresses $\tau_{1}$ and $\tau_{2}$.

Equation 2 is an expression for the sum $\tau_{s}$ of yield stresses of the two materials that gives an unstable upper bound system for a given difference in density $\Delta \rho$ and geometry. The terms $k_{U 1}, k_{U 2}$ and $k_{U 3}$ represent three different contributions to the stability of the system. Specifically, $k_{U 1}$ accounts for the energy needed to activate the internal slip surfaces and to deform the radial shear zones, $k_{U 2}$ captures the effects of friction at the walls, and $k_{U 3}$ accounts for the impact of the stress at the interface between the two materials on the stability of the system.

\subsection{Lower bound model}

A lower bound model is developed by defining an equilibrium stress state that does not violate the yield condition of the two materials. A simplified stress state that accounts for the effects of friction at the wall and at the interface between the materials (see Figure 2b) was assumed. The friction stresses at the point of slip correspond to the kinematic mechanism reported in Figure $2 \mathrm{a}$ and are applied as distributed loads $p_{1}$ and $p_{2}$ across the thickness of the columns, where:

$$
p_{1}=\frac{f \tau_{1}+\tau_{\text {min }}}{b} ; \quad p_{2}=\frac{f \tau_{2}+\tau_{\text {min }}}{b}
$$

It is thus assumed that replacing the friction stresses acting on the upper part of the system with an equivalent distributed load does not significantly affect the solution. Under these assumptions, the defined equilibrium stress state presents a vertical stress discontinuity at the interface of the two materials. The vertical and horizontal directions are the directions of the principal stresses. To obtain a lower bound solution the materials are assumed to reach their yield strength in shear at the base of the system. Writing the horizontal equilibrium for two elements A and B at the base of the columns (see Figure 2b) leads to: 


$$
\tau_{s}=m_{L} \Delta \rho
$$

where:

$$
\begin{aligned}
& m_{L}=\frac{g}{k_{L 1}+k_{L 2}+k_{L 3}} \\
& k_{L 1}=\frac{2}{h} ; \quad k_{L 2}=\frac{f\left(\frac{h}{b}-\frac{1}{\sqrt{2}}\right)}{h} ; \quad k_{L 3}=\frac{\frac{2 \alpha}{\alpha+1}\left(\frac{h}{b}-\frac{1}{\sqrt{2}}\right)}{h}
\end{aligned}
$$

Equation 6 expresses the sum $\tau_{s}$ of yield stresses of the two materials that gives a stable lower bound system for a given difference in density $\Delta \rho$ and geometry.

The two models described by Equations 2 and 6 suggest that for a given geometry and set of boundary conditions, the stability of the system can be controlled by varying the sum $\tau_{s}$ of the yield stresses of the materials and their difference in density $\Delta \rho$. Specifically, the two models indicate that for a given geometry, fresh state deformations of the system decrease with increasing stability coefficient $m$, defined as:

$$
m=\frac{\tau_{s}}{\Delta \rho}
$$

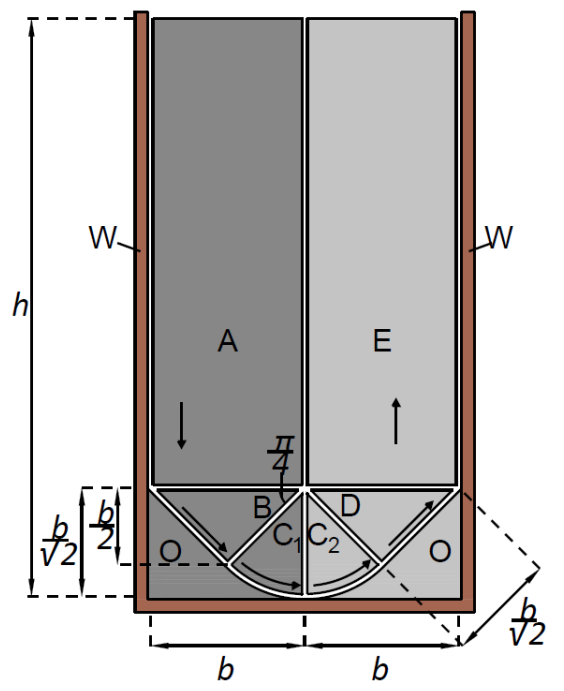

a)

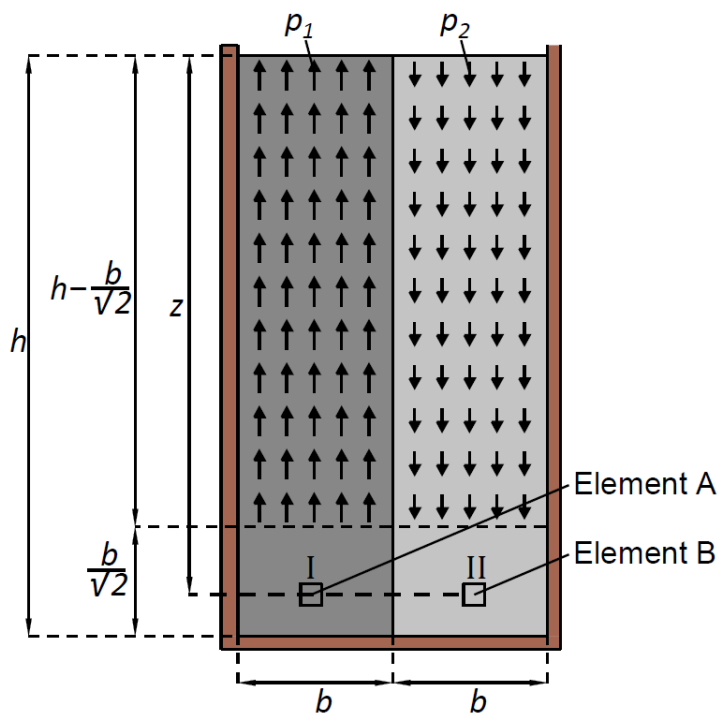

b)

Figure 2. a) Mechanism and b) Equilibrium stress state

\section{Validation against experiments}


The bound models presented in section 3 have been validated against a set of experiments on two vertical layers with tailored cementitious mixes having various yield stresses and densities. The reference tests are reported in $[10,11]$. In these experiments, a system having a column width of $b=80 \mathrm{~mm}$ and a height of $h=300 \mathrm{~mm}$ was considered. Twelve tests were performed across 24 mix compositions with a wide range of densities and yield stresses. The density of the materials was varied by adding foam to the mixes, while the yield stress was varied by altering the water content and using a polycarboxylate ether (PCE) superplasticizer. The mixes were designed to achieve sums of the yield stresses ranging from 60 to $248 \mathrm{~Pa}$ and differences in densities of up to $378 \mathrm{~kg} \mathrm{~m}^{-3}$. This led to stability coefficients $m$ ranging from 0.18 to $1.28 \mathrm{~Pa} \mathrm{~m}^{3} \mathrm{~kg}^{-1}$. As an indicator of the stability of the system, the maximum horizontal displacement $d$ of the interface between the two layers was measured in each test. A full description of the experimental methods is reported in [11].

Equations 3 and 7 were used to calculate an upper and a lower bound stability coefficient. A friction coefficient of $f=1$ was considered, representing no-slip boundary conditions at the wall. Coefficients $\alpha=0.83$ and $\alpha=0.22$ were selected for the upper and lower bound respectively. Such values represent the maximum and minimum coefficients $\alpha$ for the mixes studied experimentally. Hence, they allow to obtain indicate bounds for all tests. The calculated upper and lower bound stability coefficients were $m_{U}=0.28 \mathrm{~Pa} \mathrm{~m}^{3} \mathrm{~kg}^{-1}$ and $m_{L}=0.48 \mathrm{~Pa} \mathrm{~m}^{3} \mathrm{~kg}^{-1}$ respectively.

Figure 3 plots the experimentally measured maximum horizontal displacement $d$ (see Figure 1.b) against the stability coefficient $m$, together with the limit lines representing the analytically calculated upper and lower bound stability coefficients $m_{U}$ and $m_{L}$. The plot suggests that, according to the formulated bound models, a relationship exists between the difference in density $\Delta \rho$ of the two materials, the sum $\tau_{s}$ of their yield stresses, and the stability of the system. The displacements decrease with an increase in the stability coefficient $m$. Specifically $d$ decreases sharply for stability coefficients $m$ up to about 0.4 $\mathrm{Pa} \mathrm{m}^{3} \mathrm{~kg}^{-1}$ and more gradually for higher values of $m$. Figure 3 shows that the formulated upper and lower bound models bracket the regions of transition from unstable to stable behaviour. 


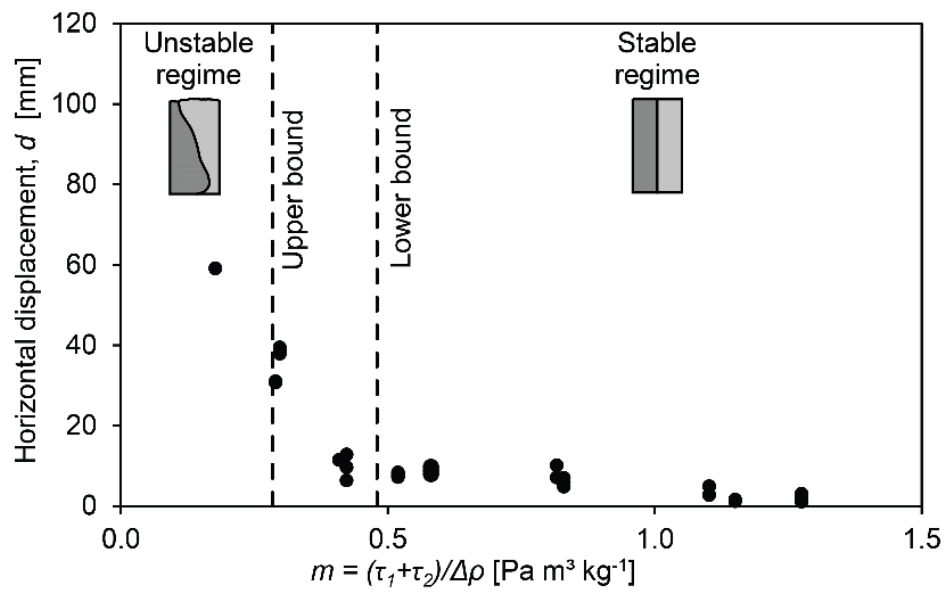

Figure 3. Maximum horizontal displacement $d$ as a function of the stability coefficient.

\section{Discussion}

Both the formulated analytical models and the experimental results suggest that, for a given system geometry, the stability of the system in the fresh state can be controlled by tailoring the material properties of the two mixes. The fresh state behaviour of the two columns is driven by the sum of the yield stresses $\tau_{s}$ and the difference in density $\Delta \rho$ between the two mixes (see Figure 3). The difference in density $\Delta \rho$ is proportional to the forces driving instability while the sum of yield stresses $\tau_{s}$ is an effective indicator of ability of the system to withstand shear stresses in the stable regime. Accordingly, the stability capacity of the system can be expressed through a stability coefficient $m$, defined here as the ratio between the sum $\tau_{s}$ of the yield stresses and the difference in densities $\Delta \rho$.

For a given geometry and set of boundary conditions, the two bound models allow for the assessment of two limit values of the stability coefficient within which plastic collapse is expected to occur. The calculated upper and lower bound solutions give an indication of the experimentally identified transition regime between stable and unstable conditions, represented by stability coefficients around $0.4 \mathrm{~Pa} \mathrm{~m}^{3} \mathrm{Kg}^{-1}$ (see Figure 3 ). This confirms that, in the case of slow flows, plasticity theory can be adopted to study the fluid behaviour of cementitious materials.

\section{Conclusions}

The following conclusions can be drawn from the present study:

- Two novel limit state models based on plasticity theory have been formulated to study the fresh state behaviour of a system composed of two vertical columns of different cementitious mixes. The models suggest that, for a given geometry and set of boundary conditions, the stability capacity of the system can be increased by 
either increasing the sum $\tau_{s}$ of the yield stresses of the materials or by minimizing their difference in density $\Delta \rho$.

- Such trends are confirmed experimentally. Specifically, it was shown that for a given geometry, fresh state deformations of the system decrease with increasing stability coefficient $m$, defined here as the ratio between the sum $\tau_{s}$ of the yield stresses and the difference in density $\Delta \rho$.

- The proposed bound models are indicative of the experimentally determined transition region between stable and unstable regimes. Thus, they can be adopted to design stable or unstable systems.

\section{Acknowledgements}

The authors would like to acknowledge the financial support of EPSRC - the Engineering and Physical Sciences Research Council (UK) [Project reference number: EP/N017668/1].

\section{References}

[1] Boden T, Andres R, Marland G. Global, Regional, and National Fossil-Fuel CO2 Emissions. Oak Ridge, Tenn., U.S.A.: Carbon Dioxide Information Analysis Center, Oak Ridge National Laboratory, U.S. Department of Energy; 2013. doi:10.3334/CDIAC/00001_V2013.

[2] Banfill PFG. Rheology of fresh cement and concrete. Rheol. Rev. 2006, The British Society of Rheology; 2006, p. 61-130.

[3] Tattersall GH, Banfill PFG. The Rheology of Fresh Concrete. Boston: Pitman Books Limited; 1983.

[4] Tattersall GH. Workability and Quality Control of Concrete. CRC Press; 2014.

[5] Wallevik OH. The Rheology of Fresh Concrete and its Application on Concrete with and without Silica Fume. 1990.

[6] Calladine CR. Plasticity for engineers. Woodhead Publishing; 2010. doi:https://doi.org/10.1533/9780857099709.93.

[7] Chen WF. Limit analysis and soil plasticity. Elsevier Science; 2013.

[8] Chen W. Plasticity, limit analysis and structural design. Int J Solids Struct 2000;37:81-92. doi:10.1016/S0020-7683(99)00079-7.

[9] Heyman J. Basic structural theory. Cambridge: Cambridge University Press; 2008. doi:10.1017/CBO9780511754487.

[10] Torelli G, Lees JM. Functionally graded concrete elements composed of vertical layers of different mixes. Proc. Guimarães IABSE Symp. 2019, Guimarães (Portugal): 2019.

[11] Torelli G, Lees JM. Fresh state stability of vertical layers of concrete. Cem Concr Res 2019;120:227-43. doi:10.1016/J.CEMCONRES.2019.03.006. 